\title{
Editorial
}

\section{Development and Historical Evolution Half a Century Ago at the Dawn of Microsurgery}

The Department of Orthopedics and Replantation Research Laboratory of the Sixth People's Hospital of Shanghai was at the forefront of technical innovation and development of limb and digital replantation and early microsurgery in the mid1960s, half a century ago. This was a period when the entirety of China was isolated from the rest of the world, with revolutionary movements spreading over the entire country. Almost unbelievably, the early development of microsurgery took place during this period, unknown to the rest of the world. As a member of the earliest research team in the replantation laboratory and a member of the surgical team in the department, the author recollects some little-known facts dating back half a century that led to nowfamiliar techniques in microsurgery.

\section{ANASTOMOSING A GREATER NUMBER OF VEINS IS NECESSARY IN DIGITAL AND ARM REPLANTATION}

Because swelling of the replanted arm was a major problem in the first cases in this unit, multiple small

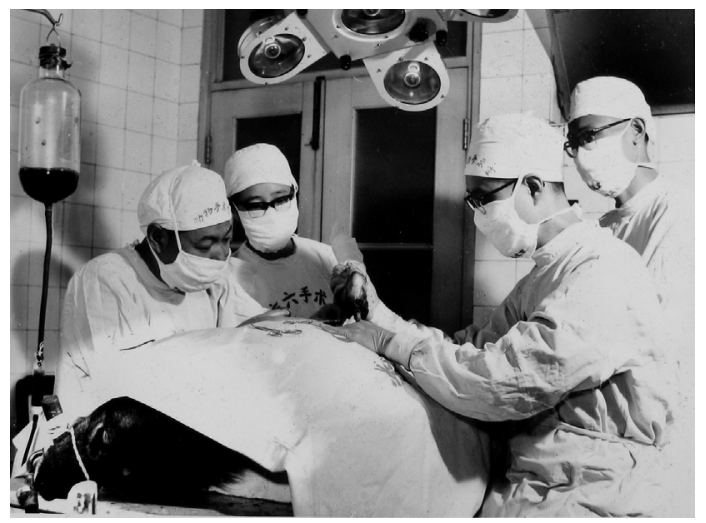

Fig. 1. The operative settings in the Replantation Research Laboratory in 1965 (on the scene from left: Zhong Wei Chen, Ling Zhang, Cheng Hua Tang, and Yong Gang Wang). (Courtesy of Cheng Hua Tang.) incisions were made to drain the venous blood. From the mid-1960s, attempts were routinely made to connect more veins than arteries in replanting an arm. Consequently, fewer replantation failures were caused by limb swelling. It became a rule to always connect more veins than arteries in limb replantation; such a rule was applied to digital replantation as well.

\section{THE ISCHEMIA TIME OF SEVERED LIMBS: COLD PRESERVATION}

Prolonging the survival time of amputated limbs or digits was a central concern of the team at that time, because patients with arm amputations often could not be transported to Shanghai from far away in a timely fashion. There were very few centers in China capable of performing limb replantation at that time. Canine experiments were carried out to test whether preservation of the amputated limb at $0^{\circ} \mathrm{C}$ to $4^{\circ} \mathrm{C}$ substantially prolonged survival of a severed limb (Figs. 1 and 2). It was found that without cold preservation, the canine limb could not be successfully replanted after 10 hours of ischemia. In contrast, cold preservation of the amputated canine limbs doubled and tripled the

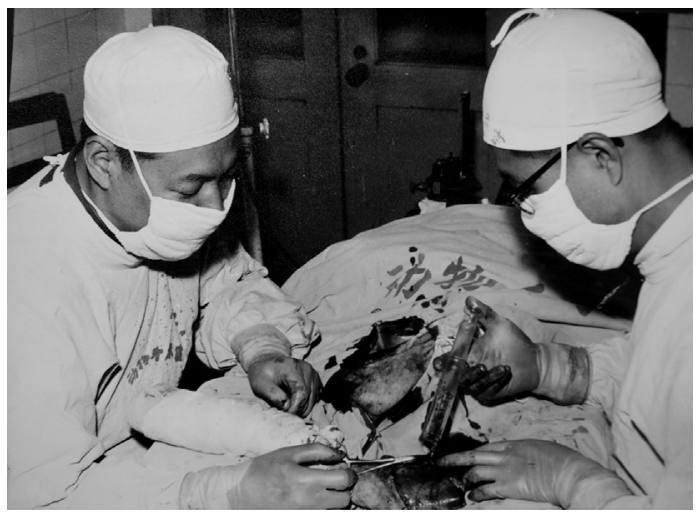

Fig. 2. Performing canine limb replantation in the research laboratory. (Courtesy of Cheng Hua Tang.)

This article is an update of a previous version originally published in Clinics in Plastic Surgery, Volume 44, Issue 2, April 2017. 


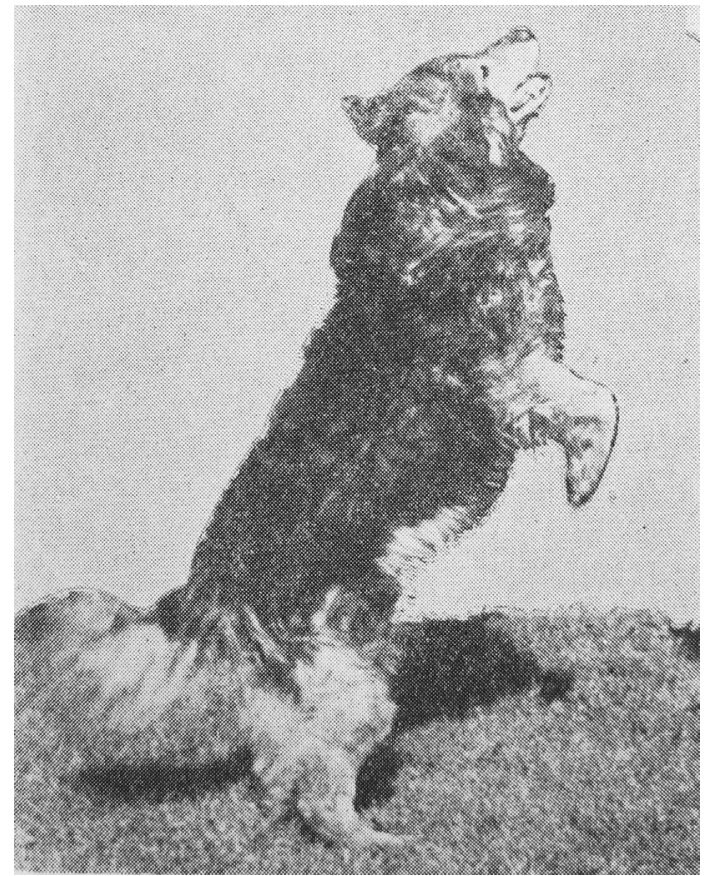

Fig. 3. The canine with a replanted hind limb after 108 hours of cold preservation following amputation.

time of ischemia allowable before a successful limb replantation. The longest time for replantation for such an amputated canine limb reached 108 hours (Fig. 3). It was thought that if cold preservation could extend the ischemia time to over 100 hours (which translates to an entire day for a human limb), there was no need to seek further solutions: cold preservation should buy enough time for any patient to be transported to a replantation center.

A funny story relates to figuring out how to achieve cold preservation in some rural areas. In 1965 , there was a phone call from a mountainous area of west Zhejiang about a farmer whose hand had been severed. How to preserve the hand for the long journey necessary? More than 100 popsicles were placed around the hand, which was wrapped in a plastic bag. After transfer to our unit 17 hours later, replantation was successful because of the cold preservation by the popsicles!

\section{RABBIT EAR AS A MODEL FOR EXPERIMENTING MICROSURGERY}

In those early days, the best model for practicing vascular anastomosis was unclear. That was when rabbit ears came into use for practicing and researching surgical replantation (Fig. 4). Vascular diameters in the rabbit ear are similar to those of vessels in the fingers, and because ear tissue is thin, vascular flow could be easily visualized against lighting. Exercise of replantation in such a rabbit ear model formed the foundation for clinical success in digital replantation in 1966 and later free vascularized muscle transfer in 1973.

\section{ANASTOMOSIS OF VESSELS UNDER $1 \mathrm{MM}$}

Higher rates of success in replanting digits were the goal in 1966, and it was thought that practicing anastomosis on vessels smaller than 1 $\mathrm{mm}$ could help. The rat femoral artery, with a diameter of $0.8 \mathrm{~mm}$, was used. Anastomoses of 100 femoral arteries were set as the goal for each member in the team. This amount of practices ensured that surgeons were quite proficient in performing clinical anastomoses of vessels in the digits.

\section{COORDINATED EFFORTS WITH MANUFACTURERS IN DEVELOPING MICROSURGICAL INSTRUMENTS}

Much effort was directed at cooperation with the Shanghai Medical Instrument Factory. At the time, there were no molds for making microsurgical needles, so workers in the factory handmade those tiny needles. One microsurgical needle usually took 2 days of continuous labor
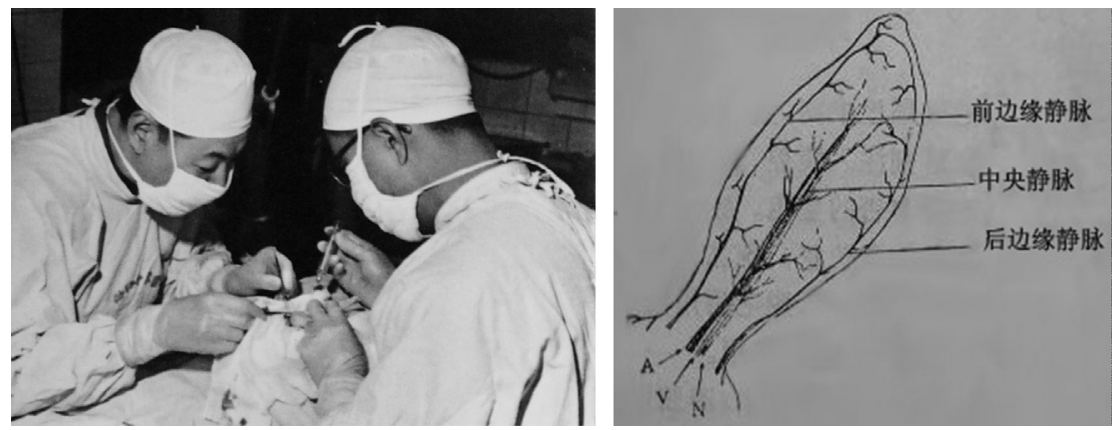

Fig. 4. Rabbit ear replantation (left) and the drawing of its arterial network (right). (Courtesy of Cheng Hua Tang.) 


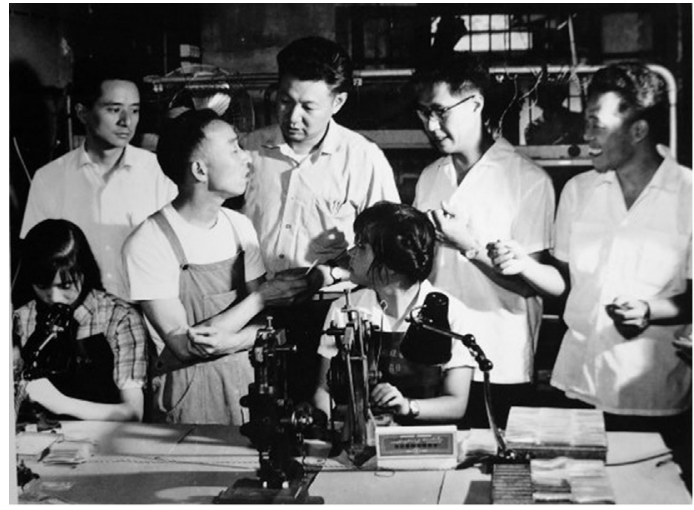

Fig. 5. In a medical instrument factory, to discuss with the workers producing microsurgical needle and sutures (back row from left: Joseph Bao, Zhong Wei Chen, Cheng Hua Tang, and Hegao He). (Courtesy of Cheng Hua Tang.)

to grind and polish under magnification. Gradually, the needle-making process was improved, and needles could be produced in batches (Figs. 5 and 6). After investigation into strength and smoothness of microvascular anastomosis with a variety of materials, nylon monofilaments were found the best material for suture of small vessels.

\section{DEVELOPING A TEST FOR THE QUALITY OF VASCULAR ANASTOMOSIS}

Driving the blood away from the vessels distal to the anastomosis site is a test of blood flow developed in replantation surgery in the mid-1960s. Immediate refilling of the vessel indicates excellent vascular anastomosis; slow or no refill of the vessels suggests poor or no blood flow. This method was verified first experimentally and then put into routine clinical use.

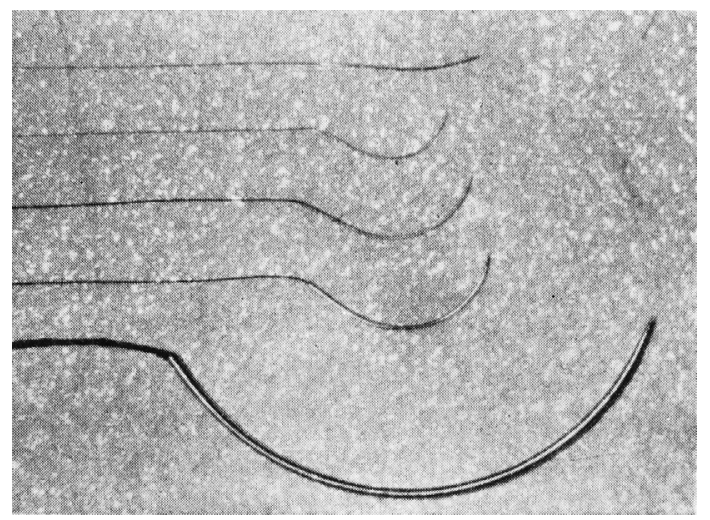

Fig. 6. Microsurgical needles and sutures (up to 11-0) made at that time. (Courtesy of Cheng Hua Tang.)

\section{HEAT AND LIGHT PROMOTE VASCULAR CIRCULATION AFTER REPLANTATION}

After a day of experimental rabbit ear replantation in 1966, we forgot to turn off the lamp used for surgery on both ears of a rabbit and left it on overnight. The ear closer to the lamp had much better vascular perfusion than the ear on the other side. This caught our attention and prompted us to consider whether heat and light might be important for successful vascular anastomosis. Consequently, we designed an experiment to determine whether heating the replanted ear produces a significant difference, and the results were positive. Those findings led to clinical use of a lamp to warm and illuminate the replanted limb or digit.

\section{ALLOGRAFT OF A CANINE LIMB}

In the winter of 1966, we started experimenting on limb allotransplantation in a canine. A hind limb was transplanted from one dog to another. The survival of the allotransplanted hind limb was 2 years, without use of any immunosuppressant. At that time, less was known about immunosuppressants, which were certainly not used. The transplanted leg survived with bony fixation, connecting muscles and tendons, and vascular anastomosis. The leg functioned well, but remarkable atrophy of the leg was noted 1 year and 9 months after surgery (Fig. 7), the leg becoming necrotic 2 years after surgery.

\section{REPLANTATION SURGERY UNDER PRIMITIVE CONDITIONS}

Replantation in the mid to late 1960s was performed under very primitive conditions: no operative microscope was available most of the time; surgical anastomosis was under simple magnification (using magnifying glasses) or with the naked eye. Vessels were anastomosed with 8-0, 9-0, $10-0$ sutures made at great cost of time by factory workers. Thus, surgeons and factory workers worked together to design and make these fine needles and sutures in amounts sufficient for use by a limited number of surgeons. Microsurgical clamps, forceps, and needle holders were all designed during that period and put into clinical use. The surgeons all knew the workers in these instrument factories well and became friends because of frequent visits to the factories. The success rate of digital replantation was $56 \%$ in the first series of replants of 151 digits with 85 digits surviving from January 1966 to December 1971 (Fig. 8), mostly using primitive microsurgical tools specially made by factory workers. 

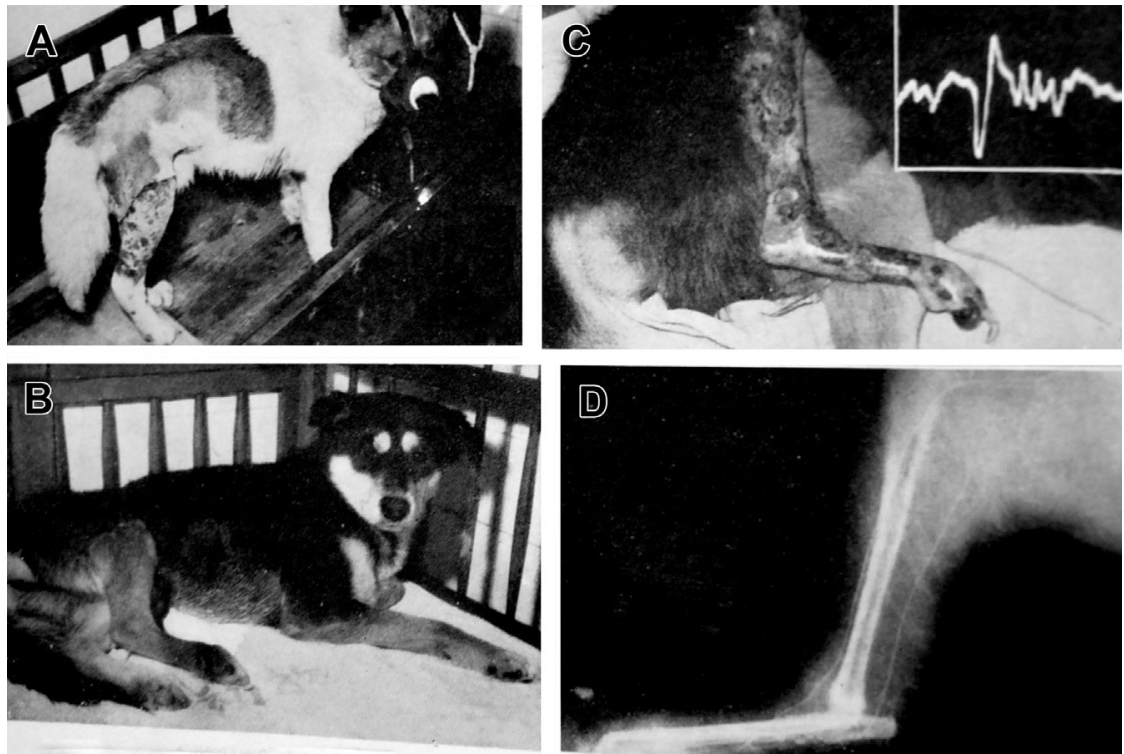

Fig. 7. Canine limb allotransplantation, survived 2 years. $(A) 2$ weeks after transplantation. $(B) 3$ weeks after surgery. (C) 1 year and 9 months after surgery. EMG examination detected muscle contraction potential, but the limb had remarkable atrophy and skin ulcerations. (D) Angiography indicated integrity of major vessels, though the limb became necrotic.

TECHNICAL PROBLEM-SOLVING AND SERVING THE PEOPLE WERE THE MAIN GOALS, WITH FEW PUBLICATIONS

As is well known, the 1960s were a period of turmoil due to the "Cultural Revolution" in China. Research institutes in many other fields of science and technology were forced to shut down.
However, fortunately, research and clinical work in replantation were strongly supported and encouraged by the government and consistently had the support of the hospital and the medical community in China as well. The Sixth People's Hospital established the Research Laboratory for Replantation of Severed Limbs in 1965, which was fully economically supported by the hospital;

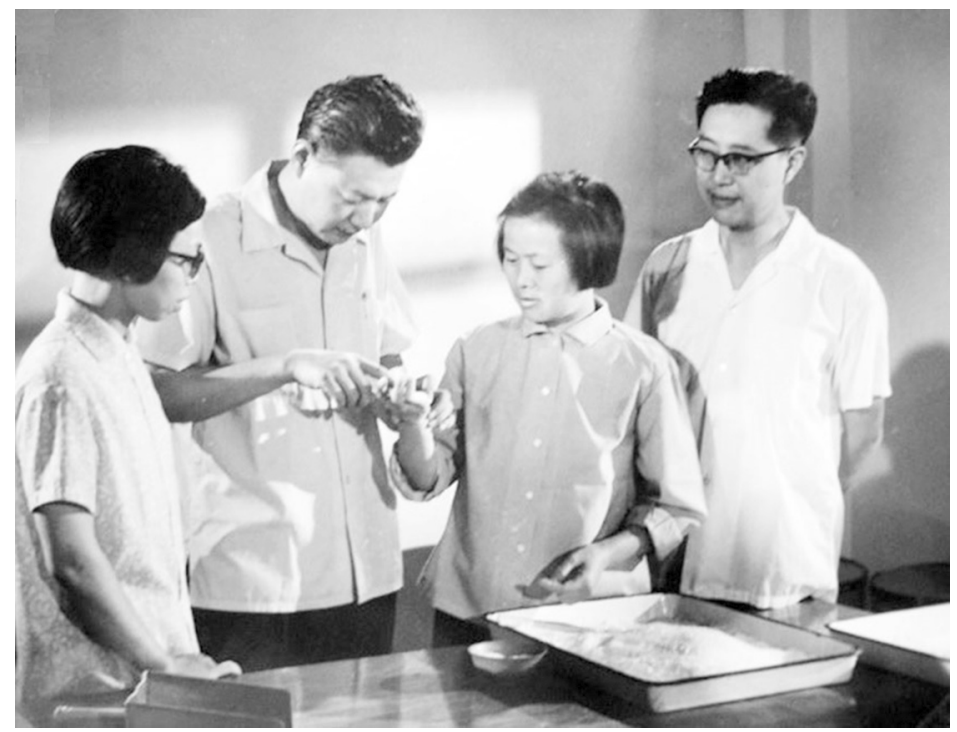

Fig. 8. Clinical follow-up of a patient after digital replantation (from left: Drs Shi Qin Shi, Zhong Wei Chen, the patient, Dr Cheng Hua Tang). (Courtesy of Cheng Hua Tang.) 


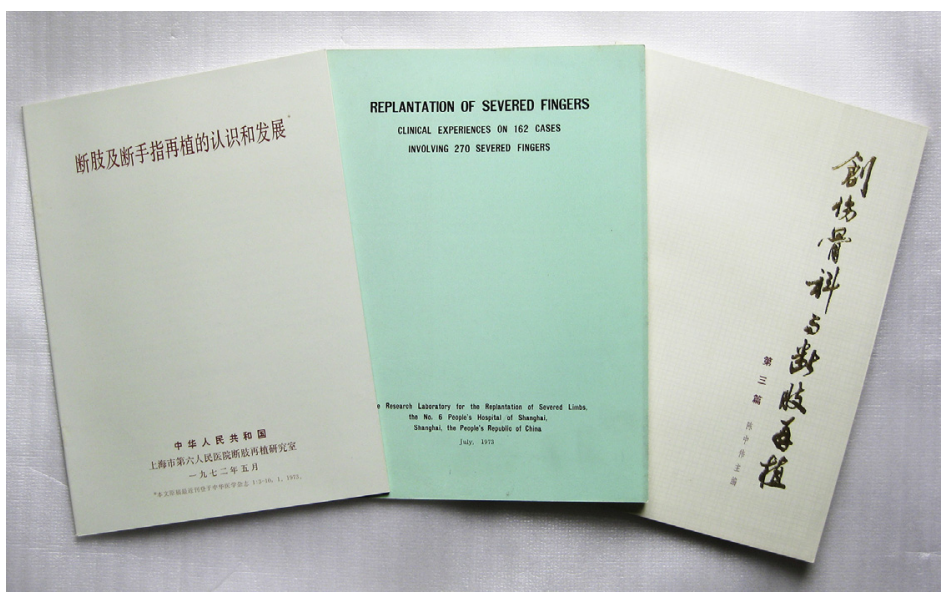

Fig. 9. Reprints of publications made at that time, without investigators' names, instead authored by "Research Laboratory for Replantation of Severed Limbs", and a book in the Chinese language. (Courtesy of Shanghai Science and Technology Press, Shanghai, China; with permission.)

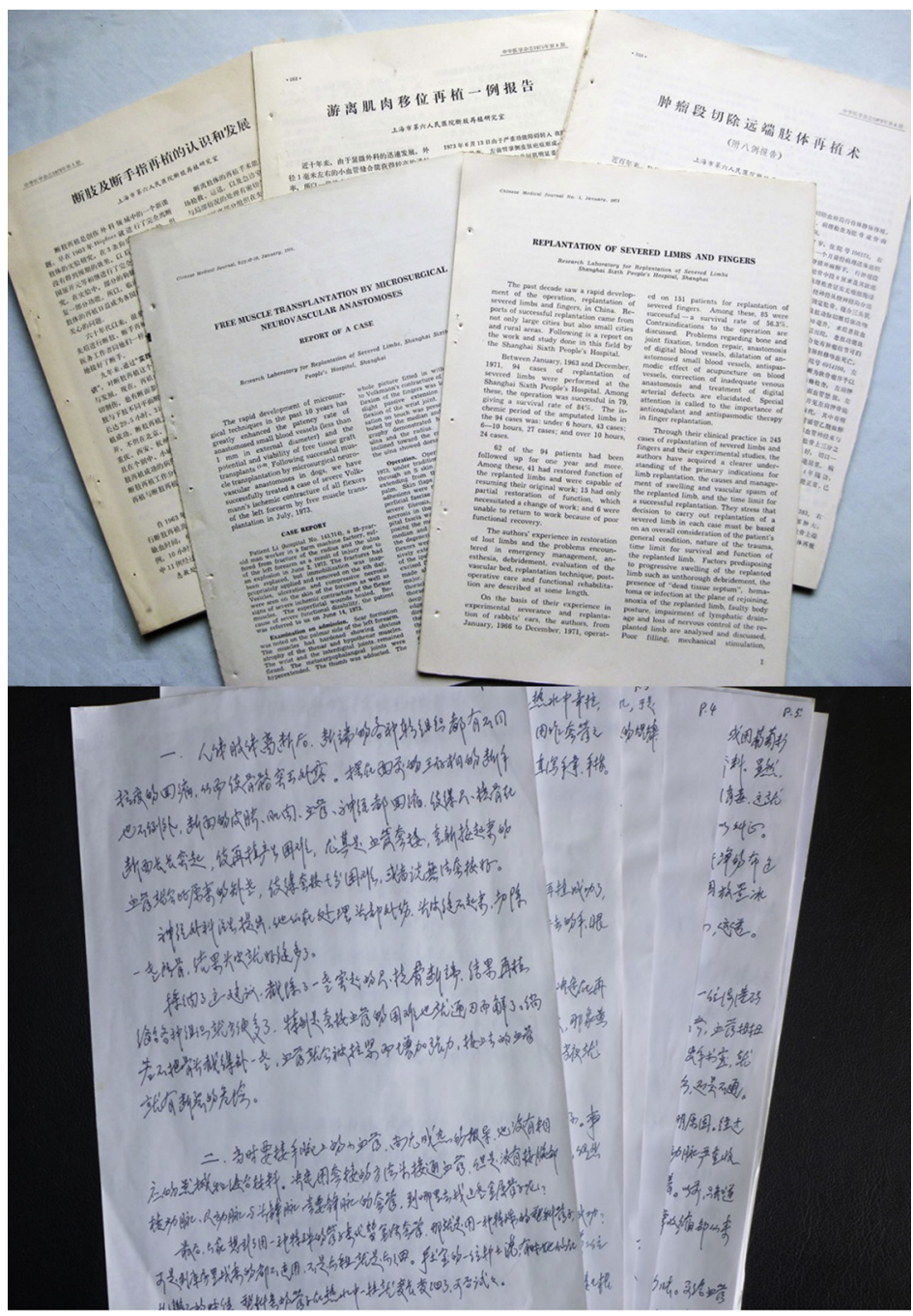

Fig. 10. The reprints of papers about clinical methods in early 1970s, authored by the "Research Laboratory" and preserved by the author (upper). The early experimental replantation and microsurgery were not published at that time, as the author recollected in a memoir about early day practice and experiments on microsurgery (lower). 


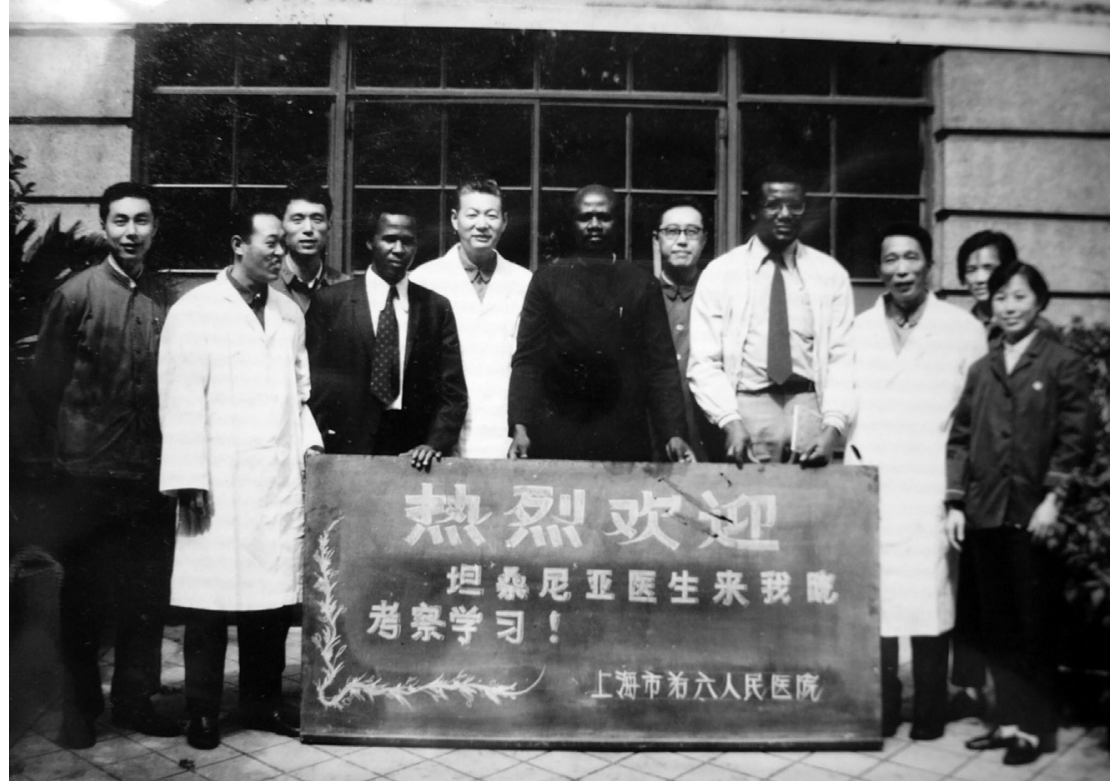

Fig. 11. Posing at the main gate of the hospital building together with a group of overseas visitors, with a welcome sign (from left: 2nd, Hegao He; 5th, Zhong Wei Chen; 7th, Cheng Hua Tang).

staff was designated, and appropriate settings were provided for experimental replantation as well as histological and biochemical assessments. The hospital had professional medical photographers take operative pictures of patients and experiments; all first-hand clinical materials were preserved. The Laboratory was located in a

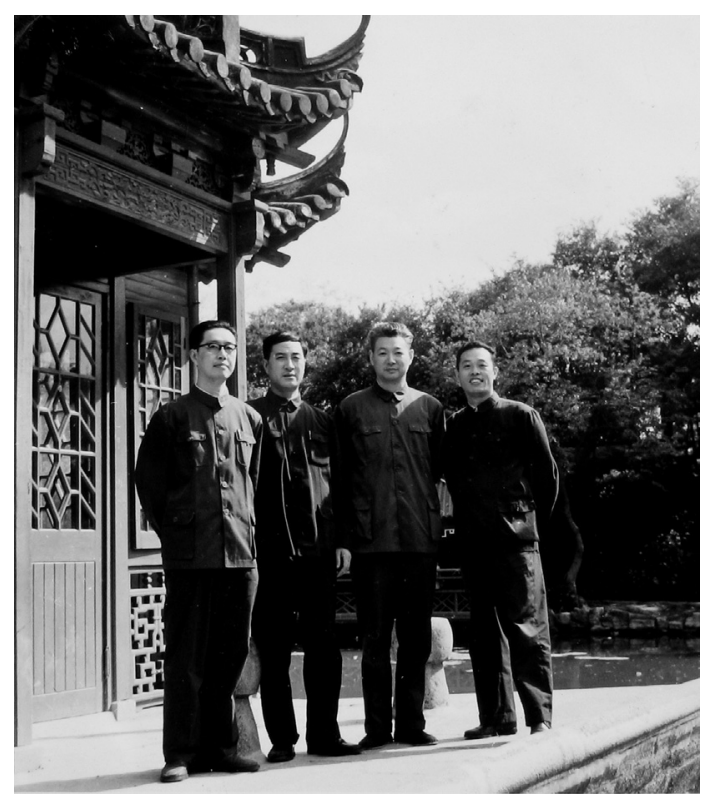

Fig. 12. A group picture taken in an outing to a suburban park of Shanghai on April 24, 1966 (from left: Cheng Hua Tang, Zhong Jia Yu, Zhong Wei Chen, and Hegao He). (Courtesy of Cheng Hua Tang.) two-story building in the hospital. We conducted investigations answering pressing clinical questions at that time and looking into fundamental techniques of microvascular surgery. The highest objective of the period was to devote oneself to the people and not to be selfish. Therefore, solving practical clinical problems was an acceptable goal. However, publications by the department and laboratory were rare, because all Chineselanguage journals virtually stopped publication, and communication with colleagues outside China did not exist. Such a harsh environment imposed many hardships and frequently interfered with work order in late 1960s. Nevertheless, surgeons were able to concentrate on solving problems encountered in replantation surgery and developing essential microsurgical techniques with little consideration of reward to themselves and no pressure to publish. Laboratory results with clinical implications were tested or simply applied directly in the clinic, followed by observation and summary of outcomes. Only a few publications were made during this period and without authors' names. Instead, they were credited to the "Research Laboratory for Replantation of Severed Limbs," and some were written but not published at all (Figs. 9 and 10). Though few or no academic conferences existed in China during the period, surgeons from all over the country and surgeons from other countries could come to learn the techniques in later years, and surgeons in the team were often invited to perform replantations in other hospitals 
in other cities, which all helped popularize the techniques of replantation and microsurgery in the field's infancy. From the 1960s to the 1970s, books on replantation and microsurgery were published in an effort to popularize the new techniques of replantation and microsurgery and later to visitors from abroad (Fig. 11).

The mid to late 1960s was a fertile period in the development of arm and digital replantation and many essential techniques that would later be called microsurgery. Microsurgery was an unfamiliar terminology that began emerging at that time, though otolaryngologists started to use operating microscopes in surgery as early as 1920s. Microvascular surgery was explored and microvascular anastomosis was experimented with and attempted in 1960s only in some regions, such as America, Japan, Australia and Europe; yet opportunities of communication were few. These teams explored this unknown field inquisitively. Only later did experience from different continents merge and complement each other. I was fortunate to be on the team led by the late Dr Zhong Wei Chen, and I recall that the relationship among the team members was intimate. Every member lived very close to the hospital. I clearly remember when Dr Chen was unable to finish surgery and go home, he gave me the keys to his apartment; my bedroom was in the hospital, where everybody could get in with common keys. They rode bicycles on the street together and went on outings to parks together during holidays (Fig. 12). I recall that one of us left a bicycle unlocked in a busy street to go to another city to operate, and came back to find the bicycle was still there days later. Though it definitely required teamwork and strenuous effort, all the team members (knowingly or unknowingly) became pioneers in microsurgical techniques, with $\mathrm{Dr}$ Chen undoubtedly the leader, foremost inventor, and greatest contributor to the field. Fifty years later, some of those on the team have already passed away. To represent the efforts of those involved, I have tried to include photographs of as many members of the early teams as possible, and share them with the members who today still treasure memories of the days of inventing and pioneering half a century ago. We all are happy to see microsurgery grow and flourish, with equipment and a scope of clinical applications unimaginable 50 years ago.

Note: Dr Cheng Hua Tang was a member of the earliest team in the Research Laboratory for Replantation of Severed Limbs (Dr Chen was the chief) and a member of the surgical team in the mid to late 1960s. With Dr Chen leading the efforts, Cheng Hua Tang arranged and executed the studies in the Laboratory in that period. He later was the vice chair of the Department of Orthopedics and chief of the Replantation Research Laboratory, Extremity Microsurgery Institute, The Sixth People's Hospital, Shanghai. In his eighties, he lives a happy life, still seeing patients three days a month. $\mathrm{He}$ is among the few early investigators of microsurgery in the 1960s still able to provide fascinating accounts of those pioneering activities started half a century ago.

Cheng Hua Tang, MD

Suite 14, Floor 6, Number 2, 120 Long, Guanlong Road, Shanghai 200061, China

E-mail address: jinbotang@yahoo.com 